\title{
Numerical calculations of the hydrodynamic performance of the contra-rotating propeller (CRP) for high speed vehicle
}

\author{
Hassan Ghassemi, Associate Prof., \\ Mohsen Taherinasab, M.Sc., \\ Amirkabir University of Technology, Tehran, Iran
}

\begin{abstract}
Several different methods have been used to analyze the marine propellers. In this paper a boundary element method (BEM) for modelling steady hydrodynamic performance of a contra-rotating propeller (CRP) has been developed. The potential flow across the front and rear propellers have been considered. By calculating the induced flow, the interaction between two propellers is studied. This method has been applied to a typical CRP. The results are included in the form of the hydrodynamic performance coefficients, total thrust and torques. Numerical calculations indicate that the predicted hydrodynamic performance shows good conformity with experimental results.
\end{abstract}

Keywords: boundary element method; contra-rotating propeller; high speed vehicle

\section{INTRODUCTION}

Because of increasing demands on higher load - carrying capacity and speed of the marine transport systems the marine research centres have concentrated their study on propeller devices that can meet this demand. However several problems arise when single propellers are used in large displacement ships or submarines to produce the required thrust. Amongst them are: increasing chance of propeller blade cavitation in heavy load conditions and vibrations due to unbalance in torque. The vibrations are transferred from the propeller to the ship body and cause instability of motion. The aforementioned problems must be considered by the designers specially in controlling rolling.

A suitable method to overcome such problems is applying the contra-rotating propellers. Such propellers are composed of two propellers which are installed on two coaxial shafts and rotate in opposite directions. Despite the disadvantages such as complexity of design and heavy weight due to the propeller's motor, the contra-rotating propellers have many advantages over single propellers, including the following:

a. generally, the rotational energy produced by the single propeller not only has no effect on the thrust but also causes loss of energy. The most important benefit of the contra-rotating propellers is their improved efficiency due to energy recovered by the back propeller. In fact, the back propeller recovers some amount of the rotational energy that is transferred to the water by the front propeller. Therefore using such propellers leads to a lower fuel consumption.

b. the torque between the two propellers is balanced, that is used to eliminate the reverse torque in devices in case when maintaining stability of motion is important.

c. the load on the blades is lower and cavitation is delayed. The lower load also allows to reach a higher power output from CRP propellers of similar diameter compared with single ones.

d. the produced noise is lower because of the uniformity of the downstream wake field of the contra-rotating propellers.

Unfortunately there is no optimized design procedure for contra-rotating propellers and the full- scale test data are not published widely.

There is no exact record of the history of contra-rotating propellers, but the first mention of them was given by Dollman and Perkins before 1800. Later, Ericsson performed experiments on model and real- scale propellers. Rota was the first who showed that the efficiency of the contra-rotating propellers is higher than the single propellers. He found that the reason for the increase in efficiency is the recovery of rotational energy transferred to water by the first propeller [1]. Several researchers performed numerical and experimental studies on the performance of the propellers and their parameters that affect it and their results were published in different papers some of which are listed by Cox et al. in [2]. Using lifting surface theory, Tsakonas et al. [3] computed the hydrodynamic forces applied on the contra-rotating propellers in stable and instable conditions. Yang $[4,5]$ used the lifting surface theory taking into account the interaction of the trailing wakes of the two propellers. Hoshino [6] also used the lifting surface theory to analyze the loads exerted onto the contra-rotating propeller shaft and has compared the results with the experimental 
data gathered by him. In another research [7], Gu and Kinnas employed the Vortex-Lattice Method (VLM) and Finite Volume Method (FVM) to study the interaction of two component propellers in contra-rotating propellers and the propeller and duct (or nozzle) in ducted propellers, based on Euler solution of the entire flow field. Ghassemi [8] carried out numerical computations of the contra-rotating propellers for two types of ships, and recently [9] on the PBCF (Propeller Boss Cup Fin) effect to propeller performance. In his research, was also made a comparison between contra-rotating and single propellers for these ships. Koronowicz [10] described the use of computer software based on Vortex-Lattice Method. In the present paper his method is modified based on the boundary element method.

As for experimental research the results of the experiments on a number of contra-rotating propellers were published by Hecker and McDonald [11]. Apart from evaluating the performance of each propeller, they also experimentally analyzed the effect of some parameters such as diameter of the front propeller, distance between propellers and pitch of the propeller. Van Manen and Oosterveld [12] performed experiments on a systematic series of contra-rotating propellers in which the front propeller had 4 blades and the rear propeller 5 blades. The results of tests performed on a number of contrarotating propellers, in which the loads applied on the contrarotating propeller in instable non-uniform and uniform flow conditions were calculated, were also published by Miller [13]. Brizzolara et al. [14] presented a full numerical lifting line model with slipstream contraction effect to determine the chord pitch and camber distribution to respect the given margins of cavitation and strength.

It is difficult to analyze a CRP in high rotational velocity field such as that of high-speed vehicle and there is no published data on such analysis using the boundary element method. In this paper, considering the physics of the problem we have obtained good results using the boundary element method. The results include the hydrodynamic performance of the rear and front propellers and their interaction. The computing of the induced velocity of the front propeller, regarded as the input velocity to the rear propeller, and the interaction of the two propellers are the most important problems which are considered in this paper.

\section{FUNDAMENTAL EQUATIONS \\ Coordinate system}

We assume that the front and rear propellers rotate with the velocity $\omega_{\mathrm{f}}$ and $\omega_{\mathrm{a}}$, respectively, in non-viscous, incompressible and irrotational flow field. The uniform flow $V_{A}$ hits the propeller upstream in axial direction.

We define the Cartesian coordinate system O-xyz (in which $\mathrm{O}$ is located at the centre of the propeller) such that $\mathrm{X}$-axis is aligned with the propellers' shaft and points downstream, $\mathrm{z}$-axis is aligned with the generator line of the key blade, and $y$-axis is so located that a counter-clockwise coordinate system is formed (Fig. 1)

In the cylindrical coordinate system, the angular position $\theta$ is measured clockwise from $z$ - axis. The coordinate $r$ is measured from $\mathrm{x}$ - axis. Therefore, the Cartesian coordinate system is transferred to the cylindrical coordinate system by using the following equation is:

$$
\left\{\begin{array}{c}
x=x \\
y=-r \sin \theta \\
z=+r \cos \theta
\end{array}\right.
$$

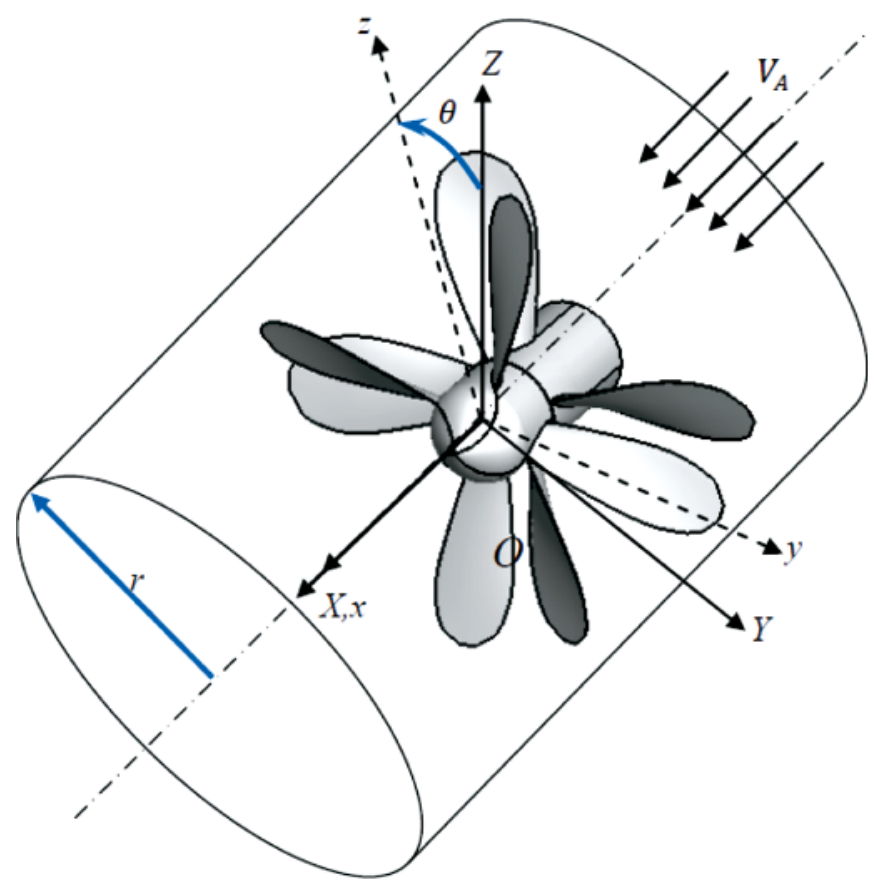

Fig. 1. Coordinate system of $C R P$

in which:

$$
\left\{\begin{array}{c}
r=\sqrt{y^{2}+z^{2}} \\
\theta=\tan ^{-1}(-y / z)
\end{array}\right.
$$

\section{Geometry of the propeller}

To construct the propeller, the geometry of the key blade of the rear propeller is formed in the Cartesian coordinate system and then transferred to the cylindrical coordinate system. Next, the Cartesian and cylindrical coordinates of other blades are obtained by using the coordinates of the key blade. For the front propeller the same procedure is repeated. As for the distance of the two propellers, the coordinates of the front propeller are displaced in axial direction.

Usually, the cross-sections of the blade at different radii in $2 \mathrm{D}$ coordinate system are known. To define the geometry of the base cross-sections, the coordinate of each point $\mathrm{P}$ on the surface of the key blade is determined. For the clockwise (CW) and counter-clockwise (CCW) propeller the following equations could be used [15]:

$$
\begin{gathered}
\mathrm{x}_{\mathrm{P}}=-\left[\mathrm{r} \tan \left(\theta_{\mathrm{R}}\right)+\mathrm{r} \theta_{\mathrm{S}} \tan \left(\beta_{\mathrm{G}}\right)\right]+\left(0.5-\mathrm{x}_{\mathrm{c}}\right) \sin \left(\beta_{\mathrm{G}}\right) \\
+\mathrm{y}_{\mathrm{u}, \mathrm{L}} \cos \left(\beta_{\mathrm{G}}\right)
\end{gathered}
$$

$\left\{y_{P}=r \sin \left[\theta_{S} \pm \frac{180\left[\left(0.5-x_{c}\right) \cos \left(\beta_{G}\right)-y_{u, L} \cos \left(\beta_{G}\right)\right]}{\pi r}\right]\right.$

$\mathrm{z}_{\mathrm{P}}=\mathrm{r} \cos \left[\theta_{\mathrm{S}} \pm \frac{180\left[\left(0.5-\mathrm{x}_{\mathrm{c}}\right) \cos \left(\beta_{\mathrm{G}}\right)-\mathrm{y}_{\mathrm{u}, \mathrm{L}} \cos \left(\beta_{\mathrm{G}}\right)\right]}{\pi \mathrm{r}}\right]$

In which, (+) applies to CCW propeller and (-) to CW one. The parameters $\beta_{\mathrm{G}}, \theta_{\mathrm{R}}$ and $\theta_{\mathrm{S}}$ are: the geometric pitch angle, rake angle and skew angle, respectively. $\mathrm{y}_{\mathrm{u}, \mathrm{L}}$ is the distance from upper and lower surfaces to the chord line, obtained as follows:

$$
\left\{\begin{array}{l}
\mathrm{y}_{\mathrm{u}}=\mathrm{y}_{\mathrm{c}}+\mathrm{y}_{\mathrm{t}} \cos \psi \\
\mathrm{y}_{\mathrm{L}}=\mathrm{y}_{\mathrm{c}}-\mathrm{y}_{\mathrm{t}} \cos \psi
\end{array}\right.
$$


where:

$\left(\mathrm{x}_{\mathrm{c}}, \mathrm{y}_{\mathrm{c}}\right)$ - camber line coordinates,

$\mathrm{y}_{\mathrm{t}}\left(=0.5 \mathrm{t}_{\left(\mathrm{x}_{\mathrm{c}}\right)}\right)-$ the half thickness of the blade,

$\check{\mathrm{r}} \quad-$ the slope of the camber line.

For obtaining the coordinates of points on other blades, we use the following mapping in which $\varphi$ is the phase angle of each blade:

$$
\left[\begin{array}{c}
\mathrm{X}_{\mathrm{P}} \\
\mathrm{Y}_{\mathrm{P}} \\
\mathrm{Z}_{\mathrm{P}}
\end{array}\right]=\left[\begin{array}{ccc}
1 & 0 & 0 \\
0 & \cos \varphi & \sin \varphi \\
0 & \sin \varphi & \cos \varphi
\end{array}\right]\left[\begin{array}{c}
\mathrm{x}_{\mathrm{P}} \\
\mathrm{y}_{\mathrm{P}} \\
\mathrm{Z}_{\mathrm{P}}
\end{array}\right]
$$

\section{Modelling the trailing vortex wake}

We assume that the surface of the wake, $\mathrm{S}_{\mathrm{w}}$, is very small and there is not any discontinuity in pressure and flow on this surface. Discontinuity in potential values is allowable. The wake geometry should be modelled so as to satisfy the Kutta condition. As mentioned earlier, in order to simplify the solution procedure the thickness of the wake is assumed equal to zero. But the edge of each cross-section has some thickness and therefore the average of the coordinates of the top and bottom surface of the blade is used to determine the wake points on the edges of the blade. The total wake propagation in the direction of the blade axis is equal to the propagation of the propeller after two complete revolutions. Experimentally, this amount of propagation is sufficient to satisfy the Kutta condition.

Changes in axial direction when the wake passes over the trailing edge of each section, are assumed negligible. It is also assumed that the propagation of each streamline of the wake is made on a constant-radius cylinder. In real situation, the radius of the trailing vortex wake (TVW) initially increases (transient region) and then decreases.

\section{The potential-based boundary element method (BEM)}

According to the made assumptions the disturbance potential satisfies the Laplace equation. Let's consider the closed region $\mathrm{V}$ with the boundary $\mathrm{S}$ (Fig. 2).

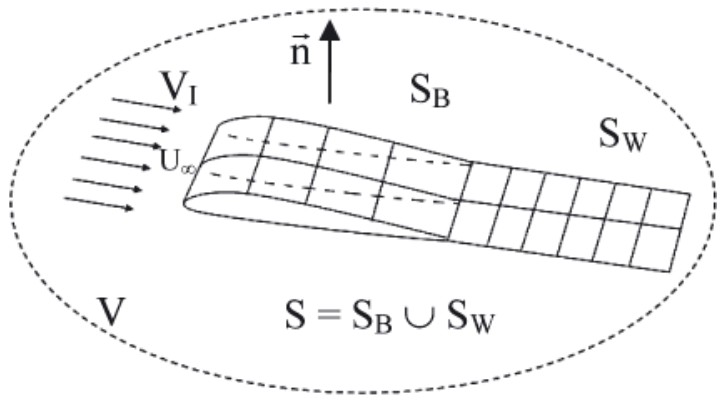

Fig. 2. Body mesh arrangement and its entering flow

The boundary value problem for the potential equation could be expressed as Neumann problem as follows:

$$
\begin{cases}\nabla^{2} \phi=0 & \text { in } \mathrm{V} \\ \frac{\partial \phi}{\partial \mathrm{n}}=\phi_{\mathrm{n}} & \text { on } \mathrm{S}\end{cases}
$$

The kinematic boundary condition which should be satisfied on the boundary $S_{B}$, is described by the following equation:

$$
\frac{\partial \phi}{\partial \mathrm{n}}=-\overrightarrow{\mathrm{V}}_{\mathrm{I}} \cdot \overrightarrow{\mathrm{n}} \text { where }: \overrightarrow{\mathrm{V}}_{\mathrm{I}}=\overrightarrow{\mathrm{V}}_{\mathrm{A}}+\vec{\omega} \times \overrightarrow{\mathrm{r}}+\overrightarrow{\mathrm{v}}_{\text {ind }}
$$

Here, $\omega$ is the propeller angular velocity of the propeller, $\mathrm{V}_{\mathrm{A}}$ is the advance velocity and $\vec{v}_{\text {ind }}$ is the induced velocity of each propeller coming from the other propeller.

To determine the vorticity the Kutta condition on the trailing edge of the blade should be satisfied. The Kutta condition is usually described as a finite velocity on the trailing edge. In other words:

$$
|\nabla \phi|_{\text {T.E. }}<\infty
$$

Considering the surface of the front and rear propellers and using Green theorem for the central point $p$ on the surface of the front and rear propellers, $\mathrm{S}_{\mathrm{B}}$, the potential problem is defined as:

$$
\begin{gathered}
2 \pi \phi(p)=\iint_{S_{B}} \phi(q) \frac{\partial}{\partial n_{q}} \frac{1}{R(p, q)} d S+ \\
-\iint_{S_{B}} \phi_{n} \frac{1}{R(p, q)} d S+ \\
+\iint_{S_{W}} \Delta \phi(q) \frac{\partial}{\partial n_{q}} \frac{1}{R(p, q)} d S
\end{gathered}
$$

$\mathrm{S}_{\mathrm{B}}$ is the sum of the surface of the front propeller, $\mathrm{S}_{\mathrm{Bf}}$, and that of the back propeller, $\mathrm{S}_{\mathrm{Bfa}}$. $\mathrm{S}_{\mathrm{W}}$ is composed of the surface of the wake of the front propeller, $\mathrm{S}_{\mathrm{Wf}}$, and that of the wake of the back propeller, $S_{\mathrm{Wa}}$. The matrix form of Eq. (9) is [16]:

$$
\begin{gathered}
{[2 \pi \mathrm{I}-\mathrm{D}]_{\mathrm{N} \times \mathrm{N}}\{\phi\}_{\mathrm{N} \times 1}=} \\
=[\mathrm{S}]_{\mathrm{N} \times \mathrm{N}}\left\{\phi_{\mathrm{n}}\right\}_{\mathrm{N} \times 1}+[\mathrm{W}]_{\mathrm{N} \times \mathrm{N}}\{\Delta \phi\}_{\mathrm{N} \times 1}
\end{gathered}
$$

Here, I is unit matrix, D, S and W are full matrices. $\phi_{\mathrm{n}}$ is determined by Eq. (7). The Kutta condition must be used for determining the unknowns $\Delta \phi$ of the doublet strength for trailing sheet vortex surface. From Eq. (10) the velocity potential $\phi$ is calculated at each element. After the velocity potential over each element is determined, velocity and pressure distributions can be calculated directly by using Bernoulli's equation:

$$
\left\{\begin{array}{l}
\mathrm{r} \\
\mathrm{V}_{\mathrm{t}}=\nabla \phi \\
\mathrm{P}=0.5 \rho\left(2 \underset{\mathrm{V}}{\mathrm{V}} \cdot \stackrel{\mathrm{r}}{\mathrm{v}} \underset{\mathrm{t}}{\stackrel{\mathrm{r}}{\mathrm{v}} \underset{\mathrm{V}}{\mathrm{r}} \cdot \mathrm{v}_{\mathrm{t}}^{\mathrm{r}}}\right)
\end{array}\right.
$$

The pressure coefficient can be expressed as:

$$
\mathrm{C}_{\mathrm{P}}=\frac{\mathrm{P}}{0.5 \rho \mathrm{V}_{\mathrm{I}}^{2}}
$$

Thrust and torque of the propellers with two components of pressure and friction are expressed as follows:

$$
\mathrm{T}=\mathrm{Z} \sum_{\mathrm{i}=1}^{\mathrm{N}} \mathrm{P}_{\mathrm{i}} \mathrm{n}_{\mathrm{xl}} \Delta \mathrm{S}_{\mathrm{i}}-\mathrm{T}_{\mathrm{Fric}}
$$

$$
\mathrm{Q}=\mathrm{Z} \sum_{\mathrm{i}=1}^{\mathrm{N}} \mathrm{P}_{\mathrm{i}}\left(\mathrm{n}_{\mathrm{yi}} \cdot \mathrm{z}_{\mathrm{1}}-\mathrm{n}_{\mathrm{zi}} \cdot \mathrm{y}_{\mathrm{i}}\right) \Delta \mathrm{S}_{\mathrm{i}}+\mathrm{Q}_{\mathrm{Fric}}
$$

where $\mathrm{T}_{\text {Fric }}$ and $\mathrm{Q}_{\text {Fric }}$ are the frictional component of thrust and torque of the propeller, respectively. They are determined by using empirical formulae proposed by ITTC. The total thrust and total torque produced by the CRP is given by:

$$
\begin{gathered}
\mathrm{T}=\left|\mathrm{T}_{\mathrm{f}}\right|+\left|\mathrm{T}_{\mathrm{a}}\right| \\
\mathrm{Q}=\left|\mathrm{Q}_{\mathrm{f}}\right|-\left|\mathrm{Q}_{\mathrm{a}}\right|
\end{gathered}
$$




\section{Hydrodynamic coefficients}

The hydrodynamic coefficients of each propeller can be obtained. For the front propeller we have:

$$
\begin{gathered}
\mathrm{K}_{\mathrm{Tf}}=\frac{\mathrm{T}_{\mathrm{f}}}{\rho \mathrm{n}_{\mathrm{f}}{ }^{2} \mathrm{D}_{\mathrm{f}}{ }^{4}}, \quad \mathrm{~K}_{\mathrm{Qf}}=\frac{\mathrm{Q}_{\mathrm{f}}}{\rho \mathrm{n}_{\mathrm{f}}{ }^{3} \mathrm{D}_{\mathrm{f}}{ }^{5}} \\
\eta_{\mathrm{f}}=\frac{\mathrm{K}_{\mathrm{Tf}}}{\mathrm{K}_{\mathrm{Qf}}} \frac{\mathrm{J}}{2 \pi} \quad, \quad \mathrm{J}=\frac{\mathrm{V}_{\mathrm{A}}}{\mathrm{n}_{\mathrm{f}} \mathrm{D}_{\mathrm{f}}}
\end{gathered}
$$

In which $\mathrm{n}_{\mathrm{f}}$ is the rotational velocity, $\mathrm{D}_{\mathrm{f}}$ is the diameter, $\mathrm{T}_{\mathrm{f}}$ is the thrust and $\mathrm{Q}_{\mathrm{f}}$ is the torque produced by the front propeller. For the rear propeller we have:

$$
\begin{gathered}
\mathrm{K}_{\mathrm{Ta}}=\frac{\mathrm{T}_{\mathrm{a}}}{\rho \mathrm{n}_{\mathrm{a}}{ }^{2} \mathrm{D}_{\mathrm{a}}{ }^{4}}, \mathrm{~K}_{\mathrm{Qa}}=\frac{\mathrm{Q}_{\mathrm{a}}}{\rho \mathrm{n}_{\mathrm{a}}{ }^{3} \mathrm{D}_{\mathrm{a}}{ }^{5}} \\
\eta_{\mathrm{a}}=\frac{\mathrm{K}_{\mathrm{Ta}}}{\mathrm{K}_{\mathrm{Qa}}} \frac{\mathrm{J}}{2 \pi}
\end{gathered}
$$

In which $\mathrm{n}_{\mathrm{a}}$ is the rotational velocity, $\mathrm{D}_{\mathrm{a}}$ is the diameter, $\mathrm{T}_{\mathrm{a}}$ is the thrust and $\mathrm{Q}_{\mathrm{a}}$ is the torque produced by the rear propeller.

Finally, as the thrust and torque of the rear and front propellers and performance coefficients of each of them are known the performance coefficients of the CRP can be obtained as follows:

$$
\begin{aligned}
\mathrm{K}_{\mathrm{T}} & =\mathrm{K}_{\mathrm{Tf}}+\mathrm{K}_{\mathrm{Ta}} \\
\mathrm{K}_{\mathrm{Q}} & =\mathrm{K}_{\mathrm{Qf}}+\mathrm{K}_{\mathrm{Qa}} \\
\eta & =\frac{\mathrm{K}_{\mathrm{T}}}{\mathrm{K}_{\mathrm{Q}}} \frac{\mathrm{J}}{2 \pi}
\end{aligned}
$$

\section{Induced velocities}

From Green's theorem, as applied to the potential field in Eq. (9), we can alternatively construct the velocity field by taking the gradient of the perturbation velocity:

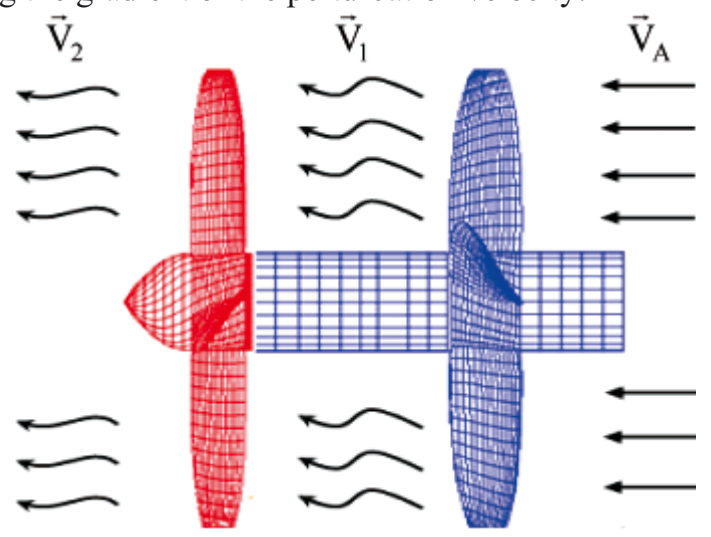

Fig. 3. Inflow velocities of $C R P$

$$
\begin{aligned}
& 4 \pi \overrightarrow{\mathrm{v}}_{\text {ind }}=\iint_{S_{\mathrm{B}}} \phi(\mathrm{q}) \nabla\left(\frac{\partial}{\partial \mathrm{n}_{\mathrm{q}}} \frac{1}{\mathrm{R}(\mathrm{p}, \mathrm{q})}\right) \mathrm{dS}+ \\
&-\iint_{S_{\mathrm{B}}} \phi_{\mathrm{n}} \nabla\left(\frac{1}{\mathrm{R}(\mathrm{p}, \mathrm{q})}\right) \mathrm{dS}+ \\
&+\iint_{S_{\mathrm{W}}} \Delta \phi(\mathrm{q}) \nabla\left(\frac{\partial}{\partial \mathrm{n}_{\mathrm{q}}} \frac{1}{\mathrm{R}(\mathrm{p}, \mathrm{q})}\right) \mathrm{dS}
\end{aligned}
$$

The induced velocities due to the front propeller, directed to the aft one, is defined by $\vec{v}_{\text {ind(FA) }}$, and vice-versa - by $\vec{v}_{\text {ind(AF): }}$. Therefore, the inflow velocity to the aft propeller is obtained as follows (Fig. 3):

$$
\left\{\begin{array}{l}
\overrightarrow{\mathrm{V}}_{1}=\overrightarrow{\mathrm{V}}_{\mathrm{A}}+\vec{\omega}_{\mathrm{f}} \times \overrightarrow{\mathrm{r}}+\overrightarrow{\mathrm{V}}_{\mathrm{ind}(\mathrm{FA})}+\overrightarrow{\mathrm{V}}_{\text {ind(AF) }} \\
\overrightarrow{\mathrm{V}}_{2}=\overrightarrow{\mathrm{V}}_{\mathrm{A}}+\vec{\omega}_{\mathrm{a}} \times \overrightarrow{\mathrm{r}}+\overrightarrow{\mathrm{V}}_{\text {ind(AF) }}+\overrightarrow{\mathrm{V}}_{\text {ind(FA) }}
\end{array}\right.
$$

The action of the rear propeller onto the front one is very low compared with the action of the front propeller onto rear one, hence it can be neglected in some cases.

\section{NUMERICAL RESULTS}

\section{Hydrodynamic performance}

In this section, the results of analysis of a typical CRP are presented. The analyzed propeller is of a 3686F-3687A type. The front propeller $(3686 \mathrm{~F})$ is a $\mathrm{CCW}$ propeller and the rear propeller (3687A) is a CW one. The geometrical parameters of the propellers are given in [13]. Main parameters of both the propellers are presented in Tab. 1. An image of the whole CRP is shown in Fig. 4. The trailing vortex wake (TVW) is shown in Fig. 5.

Tab. 1. Main dimensions of the CRP

\begin{tabular}{|c|c|c|}
\hline Parameters & $\mathbf{3 6 8 6 F}$ & $\mathbf{3 6 8 7 A}$ \\
\hline Blade number & 4 & 4 \\
\hline Diameter $[\mathrm{m}]$ & 0.3052 & 0.2991 \\
\hline P/D at $0.7 \mathrm{R}$ & 1.0 & 1.33 \\
\hline EAR & 0.303 & 0.324 \\
\hline Rotation & Anticlockwise & Clockwise \\
\hline Blade section & NACA66 $\mathrm{a}=0.8$ & NACA66 $\mathrm{a}=0.8$ \\
\hline
\end{tabular}

Run of the hydrodynamic coefficients of the front propeller $(3686 \mathrm{~F})$ is shown in Fig. 6. and for the rear propeller - in Fig. 7. The coefficients for both the propellers (i.e. whole CRP), compared with experimental results, are shown in Fig. 8. The numerical results show good agreement with the experimental data.

In 2003, Gu and Kinnas [6] analyzed this type of propeller (3686F-3687A) using coupling vortex lattice and finite volume methods. In Fig. 9 is presented a comparison made between results of the present study, Gu and Kinnas research and experimental one. It can be observed that the results of the present study are within a small error range, hence rather acceptable.

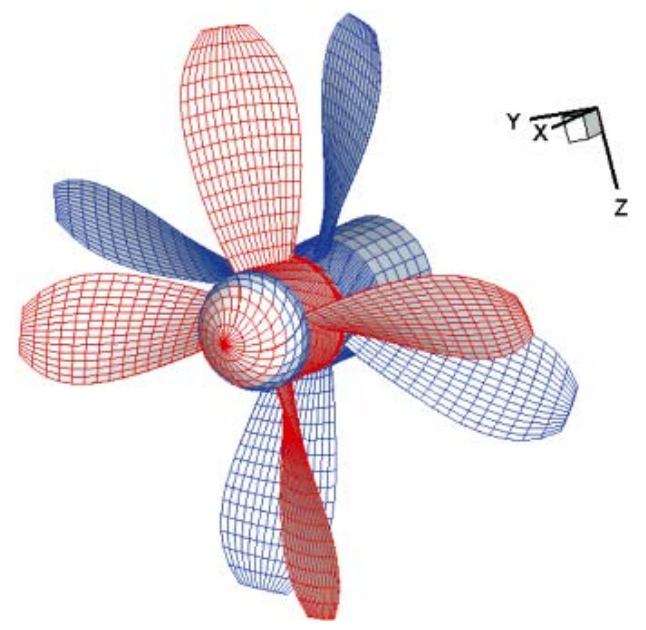

Fig. 4. $3686 F-3687 A$ CRP model 


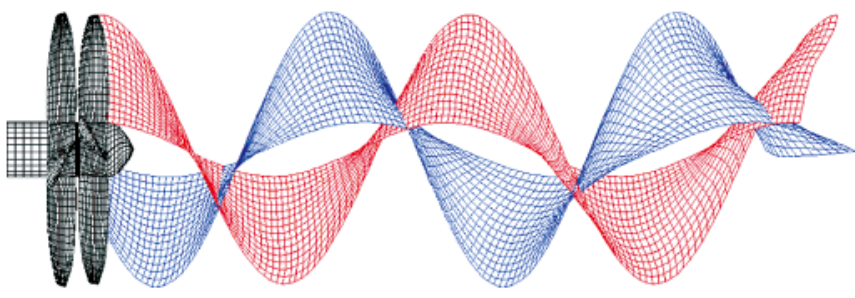

Fig. 5. TVW of the $3686 F-3687 A$ CRP model

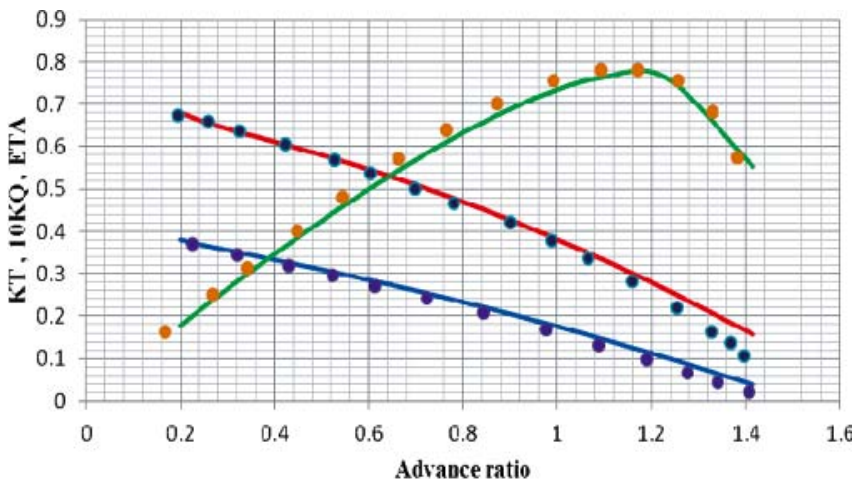

$\begin{array}{lll}\text { KTFore-Present study } & \text { 10KQFore-Fresent sudy } & \text { ETAFore-Presenr study } \\ \text { - KT-EXP } & \text { 10KQ-EXP } & \text { ETA-EXP }\end{array}$

Fig. 6. Hydrodynamic coefficients of the front propeller

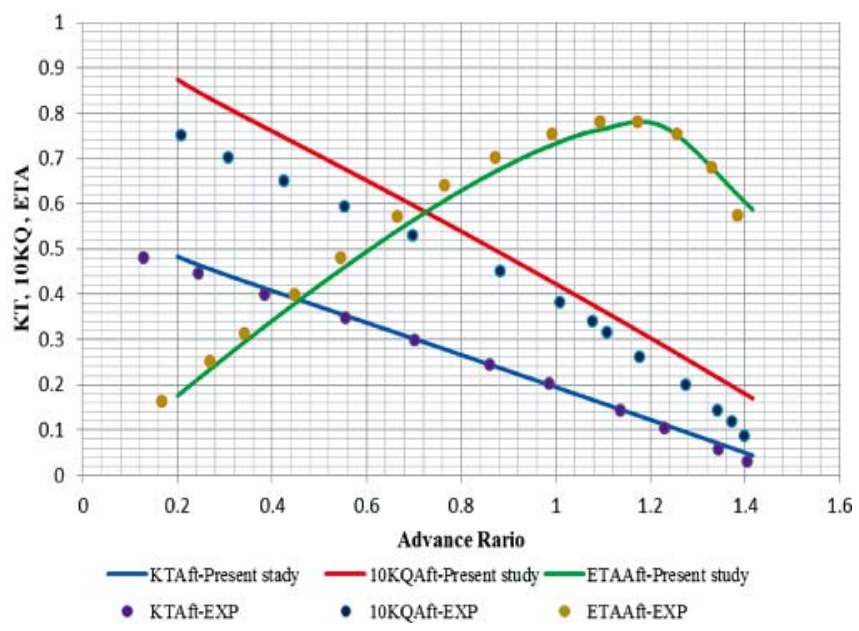

Fig. 7. Hydrodynamic coefficients of the rear propeller

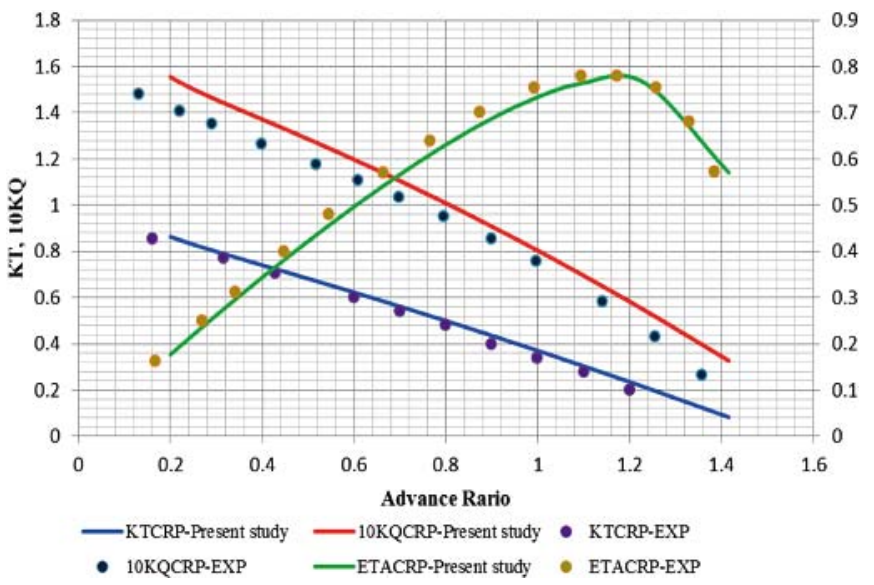

Fig. 8. Hydrodynamic coefficients of the 3686F-3687A CRP model

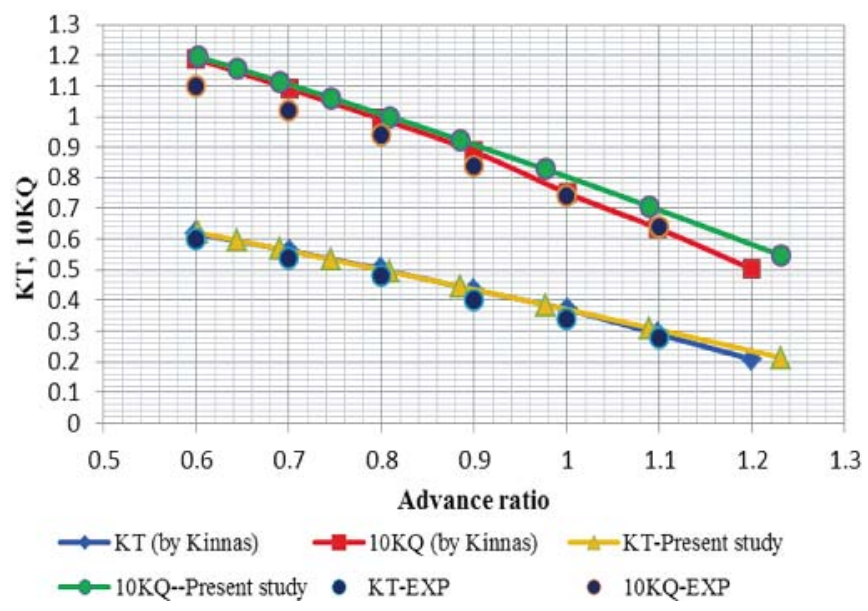

Fig. 9. Comparison of the numerical results (of the present study and Kinnas [6]) and experimental data for the considered CRP

\section{Calculations of the thrust and torque}

Values of the ratio of the thrust produced by the front propeller and the rear one related to the total thrust at the relative radius $r / R=0.7$ are obtained, respectively, as follows:

$$
\begin{gathered}
\text { T.R } \mathrm{R}_{\text {Fore }}=\frac{\text { Thrust }_{\text {Fore }}}{\text { Thrust }_{\text {total }}}=47.27 \% \\
\text { T.R }_{\text {Aft }}=\frac{\text { Thrust }_{\text {Afte }}}{\text { Thrust }_{\text {total }}}=52.73 \%
\end{gathered}
$$

It can be seen that the thrust produced by the rear propeller is higher. This may be due to the effect of the front - propeller - induced wake on the rear propeller.

Fig. 10 and 11 illustrate the thrust-RPM, torque-RPM, total thrust-RPM and total torque-RPM relations, respectively. Vectors of the torque produced by the front and rear propellers are directed against each other and thus the total torque are much lower than the individual torque of each propeller.
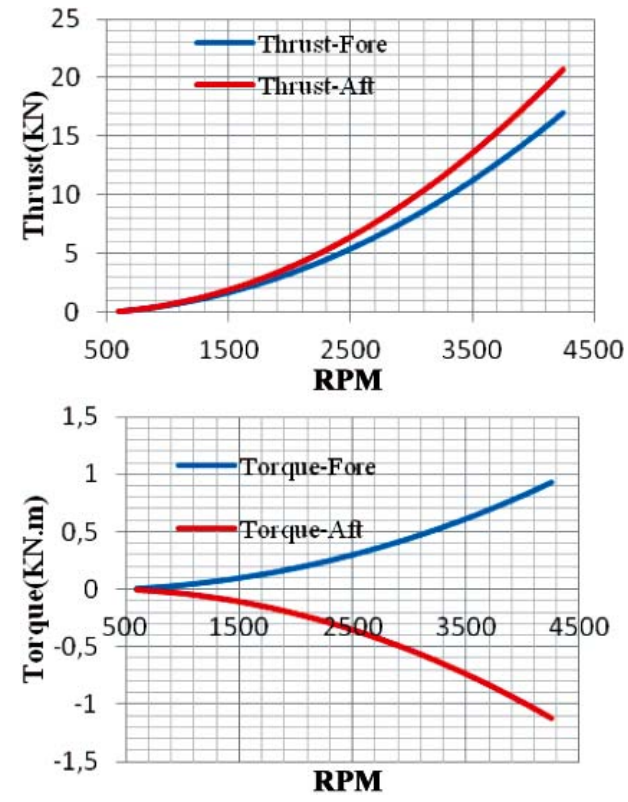

Fig. 10. Individual thrust and torque of each propeller versus propeller rotational speed (RPM) 

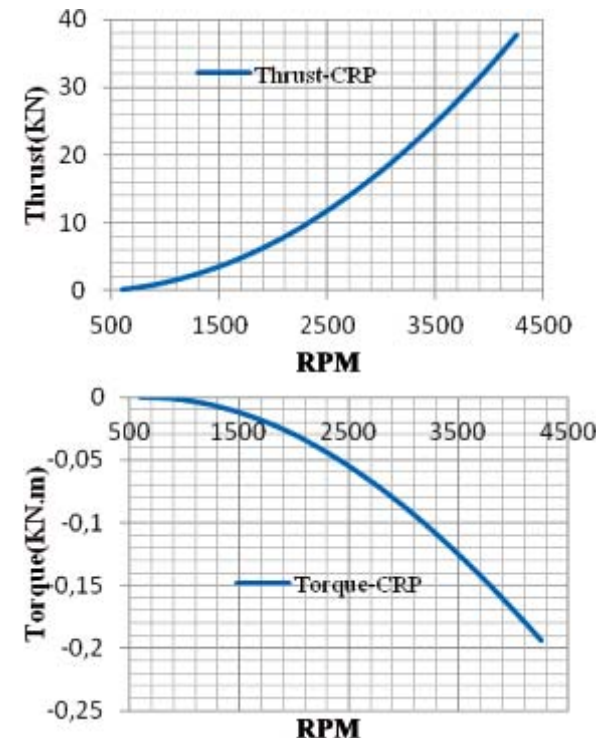

Fig. 11. Total thrust and torque of the whole CRP versus propeller rotational speed $(R P M)$

\section{Pressure distributions on blades and induced velocities}

The coefficient of contour pressure distribution on the propeller blade surfaces at $\mathrm{J}=1.19$ are shown in Fig. 12 and 13. Red colour shows the high pressure face side and blue one indicates the low pressure back side. The induced velocities are very important because the interactions between two propellers are caused by them. The axial induced wake velocity between two propellers and that behind the rear propeller is shown in Fig. 14 and 15, respectively.
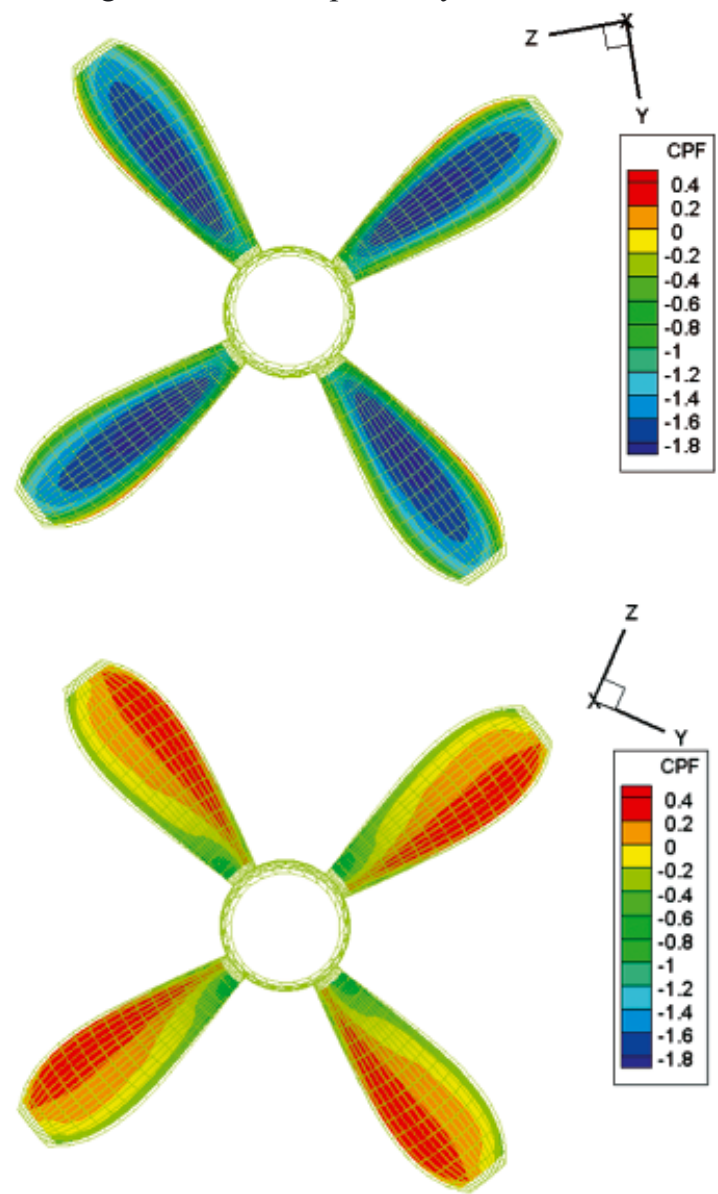

Fig. 12. Pressure distributions on the back (upper or left?) and face (lower or right?) surface of the front propeller

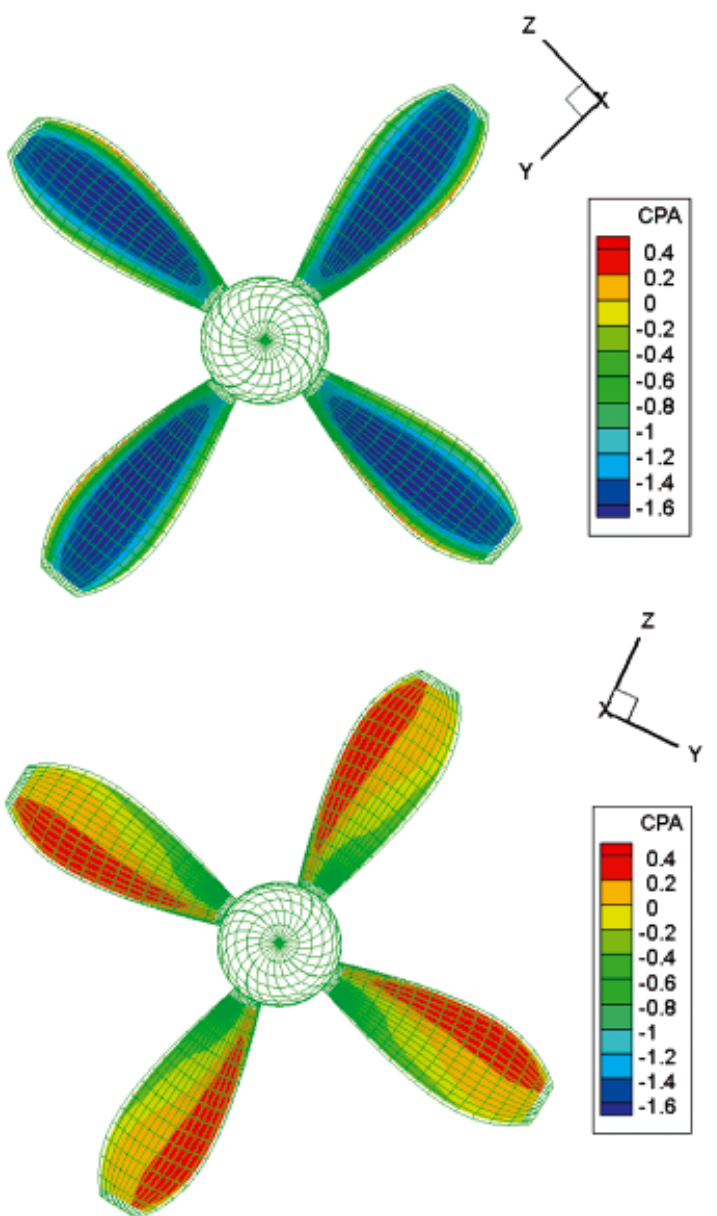

Fig. 13. Pressure distributions on the back (upper or left?) and face (lower or right?) surface of the rear propeller

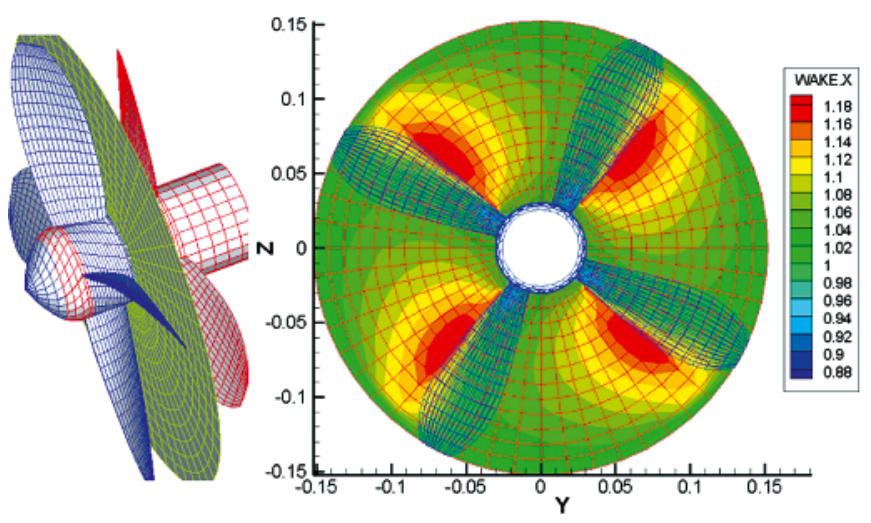

Fig. 14. Axial induced wake velocity between two propellers $(X / R=0.22)$

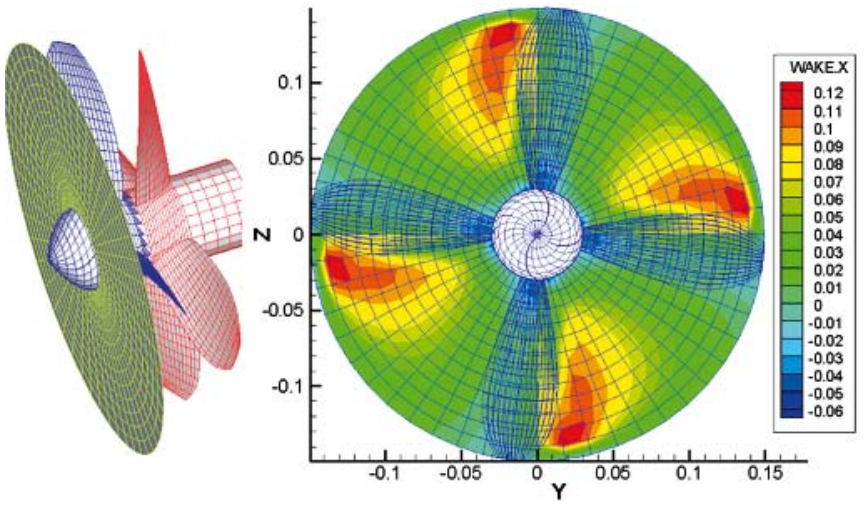

Fig. 15. Axial induced wake velocity behind the rear propeller $(X / R=0.34)$ 


\section{CONCLUSIONS}

With regard to the numerical results of the $3686 \mathrm{~F}-3687 \mathrm{~A}$ CRP calculations the following conclusions can be offered:

- As for the hydrodynamic characteristics of the front and rear propellers, it could be mentioned that the thrust and torque hydrodynamic coefficients for the rear propeller are higher than those for the front one. This is due to the effect of the front propeller wake.

- The hydrodynamic performance parameters of the CRP were obtained by superposition of the parameters of individual propellers. This is consistent with the experimental results.

- The CRP minimizes the torque produced by the propeller; hence it is recommended to apply it to devices for which elimination of roll movement is important (e.g. torpedoes). Also eliminating the torque causes less vibration to be transmitted to the ship body and thus increases the stability of motion of the ship.

- As for the pressure distribution on the rear and front propellers, it could be concluded that in a CRP the load is divided between front and rear propellers. Each propeller has its own contribution in producing the total thrust. Therefore using this system results in smaller loads on each propeller and consequently more uniform pressure distribution on the blades. Therefore in contra- rotating propellers chance of cavitation appears lower.

\section{NOMENCLATURE}

\begin{tabular}{|c|c|}
\hline $\mathrm{D}$ & - propeller diameter \\
\hline $\mathrm{D}_{\mathrm{f}}$ & - fore propeller diameter \\
\hline $\mathrm{D}_{\mathrm{a}}$ & - aft propeller diameter \\
\hline EAR & - expanded area ratio \\
\hline Z & - number of blades \\
\hline $\mathrm{J}$ & - advance velocity ratio \\
\hline $\mathrm{P} / \mathrm{D}$ & - propeller pitch \\
\hline $\mathrm{K}_{\mathrm{Tf}}$ & - thrust coefficient of the fore propeller \\
\hline $\mathrm{K}_{\mathrm{Ta}}$ & - thrust coefficient of the aft propeller \\
\hline $\mathrm{K}_{\mathrm{T}}$ & - thrust coefficient of the propeller \\
\hline $\mathrm{K}_{\mathrm{Of}}$ & - torque coefficient of the propeller \\
\hline $\mathrm{K}_{\mathrm{Qa}}$ & - torque coefficient of the propeller \\
\hline $\mathrm{K}_{\mathrm{Q}}$ & - torque coefficient of the propeller \\
\hline $\mathrm{P}$ & - pressure \\
\hline $\mathrm{C}_{\mathrm{P}}$ & - pressure coefficient \\
\hline $\mathrm{S}_{\mathrm{W}}$ & - trailing wake surface \\
\hline $\mathrm{S}_{\mathrm{B}}$ & - blade surface \\
\hline $\mathrm{T}$ & - total thrust \\
\hline $\mathrm{T}_{\mathrm{f}}$ & - fore propeller thrust \\
\hline $\mathrm{T}_{\mathrm{a}}$ & - aft propeller thrust \\
\hline $\mathrm{T}_{\text {Fric }}$ & - frictional component of thrust \\
\hline Q & - total torque \\
\hline $\mathrm{Q}_{\mathrm{f}}$ & - fore propeller torque \\
\hline $\mathrm{Q}_{\mathrm{a}}$ & - aft propeller torque \\
\hline $\mathrm{Q}_{\text {Fric }}$ & - frictional component of toque \\
\hline $\mathrm{V}_{\mathrm{A}}$ & - advance velocity \\
\hline $\overrightarrow{\mathrm{V}}_{1}, \overrightarrow{\mathrm{V}}_{2}$ & - downstream velocities of the propellers \\
\hline $\overrightarrow{\mathrm{V}}_{\mathrm{I}}$ & - inflow velocity to the propeller \\
\hline $\overrightarrow{\mathrm{v}}_{\mathrm{t}}$ & - tangential induced velocity \\
\hline $\overrightarrow{\mathrm{V}}_{\text {ind }}$ & - induced velocity \\
\hline $\overrightarrow{\mathrm{V}}_{\mathrm{ind}}(\mathrm{FA})$ & - induced velocity by fore propeller to aft propeller \\
\hline $\overrightarrow{\mathrm{V}}_{\text {ind(AF) }}$ & - induced velocity by aft propeller to fore propeller \\
\hline $\mathrm{D}, \mathrm{S}, \mathrm{W}$ & - potential matrix coefficients \\
\hline $\mathrm{n}_{\mathrm{f}}$ & - fore propeller rotating \\
\hline $\mathrm{n}_{\mathrm{a}}$ & - aft propeller rotating \\
\hline
\end{tabular}

$\vec{n} \quad-$ normal vector of the surface

$\mathrm{y}_{\mathrm{u}, \mathrm{L}}-$ distance from upper and lower surfaces to the chord line

$\left(\mathrm{x}_{\mathrm{c}}, \mathrm{y}_{\mathrm{c}}\right)$ - camber line coordinates

$\mathrm{t}_{\left(\mathrm{x}_{\mathrm{c}}\right)} \quad-$ half thickness of the blade

$\omega_{\mathrm{f}} \quad-$ angular velocity of fore propeller

$\omega_{\mathrm{a}} \quad-$ angular velocity of aft propeller

$\beta_{\mathrm{G}} \quad-$ geometric pitch angle

$\theta \quad$ - rotating angle of blade

$\theta_{\mathrm{R}} \quad-$ rake angle

$\theta_{\mathrm{S}} \quad-$ skew angle

$\varphi \quad-$ phase angle of each blade

$\psi \quad-$ camber line slope

$\phi \quad-$ velocity potential

$\phi_{\mathrm{n}} \quad-$ normal derivative of potential

$\Delta \phi \quad-$ difference potential at trailing edge

$\phi_{\text {in }} \quad-$ inflow velocity potential

$\Delta \mathrm{S}_{\mathrm{i}} \quad$ - surface area of each element

$\eta \quad-$ propeller efficiency

$\eta_{\mathrm{f}} \quad-$ fore propeller efficiency

$\eta_{\mathrm{a}} \quad-$ aft propeller efficiency

\section{BIBLIOGRAPGHY}

1. Rota, G.,: The Propulsion of Ships by Means of ContraryTurning Screws on a Common Axis. Transactions of the Institute of Naval Architects, Vol. 51, (1909).

2. Cox, B. D. and Reed, A. M.,: Contra-rotating propellers - design theory and application. In Propellers/Shafting ' 88 Symposium, Virginia Beach, VA. (1988).

3. Tsakonas, S., Jacobs, W.R., Liao, P.: Prediction of steady and unsteady loads and hydrodynamics forces on contra-rotating

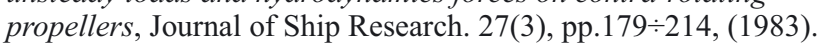

4. Yang C. J., Tamashima M., Wang G. Q., Yamazaki R.,: Prediction of the steady performance of contra-rotating propellers by lifting surface theory, Transactions of the WestJapan Society of Naval Architects No. 82, (1991).

5. Yang C. J., Tamashima M., Wang G. Q., Yamazaki R., Koizuka H.,: Prediction of the unsteady performance of contra-rotating propellers by lifting surface theory, Transactions of the WestJapan Society of Naval Architects No. 83, (1992).

6. Hoshino, T.,: Experimental and Theoretical analysis of propeller shaft forces of contra-rotating propellers and correction with full scale data, Propeller/Shafting'94 Symposium, Society of Naval Architects and Marine Engineers, Virginia Beach, (1994).

7. Gu, H., Kinnas, S.A.,: Modelling of contra-rotating and ducted propellers via coupling of a vortex-lattice with a finite volume method. Propellers/Shafting 2003 Symposium, Society of Naval Architects and Marine Engineers, Virginia Beach (2003).

8. Ghassemi, H.: Hydrodynamic performance of coaxial contrarotating propeller (CCRP) for large ships, Polish Maritime Research (59), Vol., 16, pp. $22 \div 28$, (2009).

9. Ghassemi, H., Mardan, A., Ardeshir, A.: Numerical analysis of hub effect on hydrodynamic performance of propellers with inclusion of PBCF to equalize the induced velocity. Polish

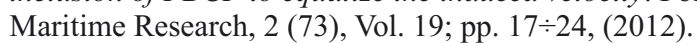

10.Koronowicz, T., Krzemianowski, Z., Tuszkowska T., Szantyr J.A.,: A complete design of ship propellers using the new computer system, Polish Maritime Research (59), Vol. 16, pp. $29 \div 34,(2009)$.

11.Hecker, R., and McDonald N. A.,: The effect of axial spacing and diameter on the powering performance of contra-rotating propellers. David Taylor Model Basin, Report 1342, Feb. (1960).

12.Van Manen J. D. and Oosterveld M. W. C.,: Model Tests on Contra Rotating Propellers. $7^{\text {th }}$ Symposium on Naval Hydrodynamics, Rome, Italy (1968).

13.Miller, M. L.,: Experimental determination of unsteady forces on contra-rotating propellers for application to torpedoes. David Naval Ship Research and Development Centre Report, SPD659-02, (1981). 
14.Brizzolara, S., Tincassi, E.P.A., Grassi, D.,: Design of contrarotating propellers for high speed stern thrusters. Ship and Offshore Structures, Publication no, 2:2, pp.169 $\div 182$, (2007).

15.Carlton J. S.,: Marine Propellers and Propulsion. Second edition, published by Elsevier Ltd, (2007).

16.Ghassemi, H., Ghadimi, P.,: Hydrodynamic efficiency improvement of the high skew propeller for the underwater vehicle under surface and submerged conditions. J. Ocean Univ. of China (JOUC), Vol. 10, pp.314 $\div 324$ (2011).

\section{CONTACT WITH THE AUTHORS}

Hassan Ghassemi, Associate Prof., Mohsen Taherinasab, M.Sc.,

Department of Ocean Engineering, Amirkabir University of Technology, Tehran, IRAN e-mail: gasemi@aut.ac.ir,

tel.: +98-21-64543112, fax: +98-21-66412495. 